\title{
\begin{tabular}{l|l} 
POLITIQUES \& & Politiques et management public
\end{tabular}
}

\section{Réformer par les outils ou par les hommes? Un bilan quantitatif de la « modernisation de la gestion » de l'Etat}

Human ressource or quality management? A quantitative approach of public administration in France

Gilles Jeannot et Danièle Guillemot

\section{OpenEdition \\ Journals}

Édition électronique

URL : http://journals.openedition.org/pmp/2926

DOI : $10.4000 / p m p .2926$

ISSN : 2119-4831

\section{Éditeur}

Institut de Management Public (IDPM)

\section{Édition imprimée}

Date de publication : 15 septembre 2010

Pagination : 73-102

ISSN : 0758-1726

Référence électronique

Gilles Jeannot et Danièle Guillemot, «Réformer par les outils ou par les hommes ? Un bilan quantitatif de la « modernisation de la gestion » de l'Etat », Politiques et management public [En ligne], Vol 27/4 | 2010, mis en ligne le 15 septembre 2012, consulté le 20 avril 2019. URL : http:// journals.openedition.org/pmp/2926 ; DOI : 10.4000/pmp.2926 


\title{
RÉFORMER PAR LES OUTILS OU PAR LES HOMMES? UN BILAN QUANTITATIF DE LA «MODERNISATION DE LA GESTION» DE L'ÉTAT
}

\author{
Gilles JEANNOT*, Danièle GUILLEMOT**
}

\begin{abstract}
Remerciements L'article repose sur l'enquête COI, changement organisationnel et informatisation, pilotée par le centre d'étude de l'emploi (CEE) et financée par la DARES et la DGAFP. La présente exploitation s'inscrit dans le programme COI-COSA soutenu par l'agence nationale de la recherche (ANR), programme " entreprises ».
\end{abstract}

Résumé

Mots-clés

L'article présente des résultats issus d'une enquête couplée employeurs/ employés concernant la diffusion des outils informatiques et de gestion et l'organisation du travail dans les administrations d'Etat. II met en évidence deux modalités de modernisation de l'administration : l'une centrée sur les engagements de prestation à destination des clients et l'autre sur certaines attitudes en matière de ressources humaines. Ces orientations sont associées à des caractéristiques du travail distinctes : mise sous contrôle de l'activité pour la première, différenciation des agents (carrière, salaire) pour la seconde. Ces modalités discrètes de la réforme marquent l'activité quotidienne des agents peut-être plus que les transformations, plus débattues, associées au contrôle des coûts et à la réforme budgétaire.

Outils de gestion, gestion des ressources humaines, qualité, technologie de l'information et de la communication.

\section{HUMAN RESSOURCE OR QUALITY MANAGEMENT? A QUANTITATIVE APPROACH OF PUBLIC ADMINISTRATION IN FRANCE}

Abstract

The paper presents results from a double survey on employers / employees in regard dissemination of computer tools and management and work organization in state administrations. It highlights the two ways of modernization of the administration: one focused on delivery commitments to customers and the other on attitudes regarding human resources. These guidelines are associated with distinct work characteristics: supervised activity for the first; differentiation between agents (career, salary) for the second. These discrete modalities of reform are more effective in the daily activity of most agents than cost control and budgetary reform transformations under the spotlights.

Keywords

Management tools, human ressource management, quality, information and communication technology.

* Chercheur LATTS, Université Paris-Est - gilles.jeannot@enpc.fr

** Chercheur Laboratoire de sociologie quantitative CREST-INSEE - daniele.guillemot@ensae.fr 
Introduction

\footnotetext{
${ }^{1}$ Les auteurs distinguent (p. 14-15) de manière emboîtée, les instruments (dispositifs techniques génériques porteurs d'une conception du rapport politique/société), les techniques (dispositifs concrets opérationnalisant l'instrument) et les outils, micro-dispositifs au sein d'une technique).
}

La diffusion de nouveaux outils et pratiques de gestion a été une dimension importante de la réforme de l'administration. Elle en a même été considérée comme le cœur par les promoteurs du courant du «management public» au tournant de 1990. Par la suite l'attention des réformateurs s'est portée sur des enjeux plus larges de compte rendu au parlement ou de réforme des structures organisationnelles, reléguant ces questions d'outils dans le clair obscur de la technique gestionnaire et des routines informatiques.

Cette relégation n'empêche pas une interrogation qui monte progressivement parmi les observateurs des réformes en cours. Elle a en particulier été réintroduite dans l'ouvrage de Lascoumes et Le Gales (2004) Gouverner par les instruments. La notion d'instrument est envisagée dans une acception beaucoup plus large que celle des outils de gestion ${ }^{1}$, mais l'ouvrage permet de dessiner deux hypothèses parallèles applicables plus spécifiquement aux outils de gestion. Les articles de Philippe Bezes (2004) et Bruno Palier (2004) suggèrent que les instruments seraient mobilisés pour mener de manière discrète et en douceur des réformes qu'il est difficile de mener frontalement. Ceci rejoint les positions de Geert Bouckaert (2003, p. 52) qui replace la mobilisation de nouveaux outils de gestion dans quatre modalités possibles de la réforme : maintenir (faire fonctionner au mieux les modes traditionnels de contrôle), moderniser (introduire de nouvelles méthodes de gestion), " marketiser » (introduire des mécanismes type marché), minimiser (confier les tâches au privé). II juge au terme d'une évaluation générale des politiques de réforme que la modernisation centrée sur la gestion représente une stratégie de réforme relativement progressive, et à ce titre efficace pour les pays d'Europe continentale.

L'article de Patrick Le Gales (2004) introduit une seconde hypothèse plus critique qui met l'accent sur une forme nouvelle de contrôle qui passerait par des outils de compte-rendu, du type tableaux de bord, illustré en l'occurrence dans le cas des relations entre l'Etat central et les collectivités locales. On retrouve là les réflexions critiques menées outre-manche sur la mise sous contrôle du travail associée au New Public Management (Hoggett, 1996) ou la lecture des réformes suisse et canadiennes par David Giauque (2003) qui y perçoit l'importation de mode de "contrôle souple» inspirées des pratiques du privé. En d'autres termes, les instruments seraient associés à une stratégie d'imposition de la réforme. Plus récemment Sylvie Trosa (2010), dans une posture plus directement liée à l'action, propose une troisième hypothèse : la mobilisation d'outils de gestion qui se substituent de fait à des "démarches" plus collective et plus élaborées aurait pour «effet pervers» de vider de son sens le projet de réforme de l'administration et de retirer toute capacité réflexive aux agents de ces administrations. Ceci rejoint un risque énoncé précédemment par Annie Bartoli (2007) pour le 
management public de retomber dans «sa pathologie bureaucratique et instrumentale ». Ce faisant cette technicisation de la réforme ne conduirait pas à son accomplissement, comme dans les deux hypothèses précédentes, mais à son échec par embourbement bureaucratique, incompréhension des exécutants et dilution du projet : les outils contre la réforme.

L'enquête changement organisationnel et informatisation (COI) de 20062007, apportant des informations statistiques couplées sur l'usage des outils de gestion et sur les caractéristiques du travail, dans l'administration de l'Etat et dans les entreprises marchandes, permet d'apprécier l'ampleur et la nature de certaines transformations gestionnaires promues par le courant du «management public » et de mesurer certains de leurs effets. Ce dispositif d'enquête offre ainsi une occasion inédite et opportune de contribuer à ce débat en appréciant le niveau de développement des outils (leur diffusion a-t-elle été freinée après que ces outils ont été moins sous le feu de projecteur ?), en mesurant la part respective de chaque type d'outils (les outils de contrôle prennent-ils le dessus sur les outils facilitant la responsabilisation ou la coopération des agents ?) et enfin en permettant de mettre en relation la mobilisation de ces outils et des transformations dans le travail des agents des administration. Le parti pris de traitement de l'enquête qui consiste à traiter à la fois les outils et les composantes de l'administration dans leur diversité, réserve la possibilité d'apporter une appréciation différenciée des hypothèses proposées dans ce débat sur les outils de gestion et la réforme de l'Etat.

\section{Les outils de gestion et la réforme administrative}

La réforme, le projet de modernisation et les outils de gestion tissent des liens variés au cours du temps. Par réforme on entendra ici tous les actes de transformation de l'intervention de l'Etat sur ses moyens et ses finalités. Par «modernisation» on entendra le projet de traiter les dérives de la bureaucratie en mettant en œuvre des méthodes alternatives de gestion ${ }^{2}$ souvent issues du secteur privé. Par outil on entendra enfin les supports codifiés des pratiques de gestion (documents comptables, normes, procédures, applications informatiques ....) (Moisdon, 1997). Les trois notions sont clairement distinctes : une bonne partie de la réforme ne porte pas sur des questions de gestion publique (par exemple la privatisation ou la réallocation de fonds entre différentes politiques ...), le projet de modernisation inclut des réflexions sur les structures de l'organisation, comme la création d'agences, qui vont au-delà de la mise en œuvre d'outils de gestion, et enfin une partie de la diffusion des outils de gestion (en

\footnotetext{
${ }^{2}$ Le choix d'un terme pour caractériser cet angle de la réforme est délicat. Le terme " modernisation " a l'avantage d'être employé le plus souvent par les promoteurs de cette orientation. L'expression « management public » a pu à un certain moment condenser cette orientation (Bartoli, 1997, p. 15). Cependant les définitions savantes de la notion refusent la restriction de l'expression à ce sens commun (Gibert, 1986). Enfin l'expression « nouvelle gestion publique » qui traduit le « new public management », inclut des principes d'action qui vont très largement au-delà de la diffusion de nouveaux instruments de gestion et en particulier qui visent à « minimiser » la sphère d'intervention publique.
} 
particulier certains outils informatiques) s'est faite en dehors des débats concernant la réforme ou d'un projet explicite de «modernisation».

La chronologie proposée par Philippe Bezes (2009) permet de situer la manière dont la question des outils de gestion a été problématisée au cours du temps. Les promoteurs de la RCB ont été beaucoup plus attachés à des outils budgétaires et des instruments de calcul économique qu'à la réflexion sur les modalités de gestion des services. Certaines initiatives de déconcentration de la gestion, de tableau de bord ou d'informatisation du traitement de dossiers relèvent cependant d'une approche plus gestionnaire (Poinsard, 1987). La période 1972-1981 est caractérisée par la mise sur agenda d'une critique de la bureaucratie, mais le répertoire d'action était alors beaucoup plus juridique que gestionnaire. Les savoirs gestionnaires et le projet d'un «management public », pourtant disponibles, seront peu mobilisés par les réformateurs de cette époque. Après la période de transition liée à l'arrivée de la gauche au pouvoir (1981-1984), la période 1984-1991, occupe une position centrale dans la promotion de nouveaux outils de gestion publique. Le gouvernement de gauche va lier réforme et management public autour du slogan de la «qualité », approche qui sera prolongée par le gouvernement Balladur.

En 1989, la circulaire sur le «renouveau du service public» signée par Michel Rocard s'approprie encore plus nettement le projet du management public. Dans ce document, les questions de gestion sont au centre de la réforme et l'accent est mis sur les modalités plus que sur les structures: cercle de qualité, projets de services, centres de responsabilité. Ces liens forts entre la réforme et les outils de gestion vont être remis en cause dans la période suivante par les rapports qui précèdent la circulaire sur la «réforme de l'Etat et des services publics» d'Alain Juppé (1995). L'enjeu premier n'est plus alors de mieux faire mais de moins faire : c'est tout le débat sur l'Etat stratège. Les solutions envisagées sont alors tout au plus organisationnelles (créations d'agences) et les instruments de gestion relégués en périphérie. Au tournant des années deux-mille, la loi organique sur les lois de finance (Lolf) remet au centre la question de la gestion, les outils associés au budget et tout particulièrement la production d'indicateurs de résultats. Les stratégies ministérielles de réforme engagées dans la foulée (premier programme en 2003) se traduisent alors par la prescription de nouvelles listes d'outils gestion ou de démarches d'informatisation ${ }^{3}$. La révision générale des politiques publiques dont l'enjeu affiché est celui de la bonne allocation des fonds distribués, éloigne de nouveau, dans son titre et ses objectifs explicites, la question de la réforme de celle de la gestion. En pratique, la RGPP met de fait l'accent sur les structures et les organigrammes plus que sur les outils.

\footnotetext{
${ }^{3}$ La question de l'e-administration a constitué un retour, vers cette époque, des logiques les plus optimistes et technicistes concernant les effets des outils en particulier sur la démocratie. Nous ne traiterons ici que de manière indirecte de ce sujet.
} 
Un regard sur la revue «politiques et management public» offre une autre manière de souligner le caractère central du tournant des années 1990 dans la problématisation conjointe de ces trois dimensions. Un dénombrement des articles consacrés à la mise en œuvre d'outils de gestion dans le secteur public fait ressortir trois moments. Depuis le lancement de la revue en 1983 jusqu'en 1988 on recense 15 articles de cette catégorie dont 10 portent sur les outils comptables ou de contrôle de gestion, les terrains d'expérimentation sont alors les collectivités locales (7) et l'hôpital (3). Le second moment va de 1989 à 1993 avec 14 articles sur des innovations de gestion. Une nouvelle rubrique est créée pour donner la parole aux acteurs de ces transformations gestionnaires. Les thèmes privilégiés sont alors les démarches de contrat ou de projet de service, de centre de responsabilité (6) et les ressources humaines (5). Les ministères ingénieurs deviennent alors le centre de l'attention $(7)^{4}$. Cette période est refermée par un numéro spécial en 1993 intitulé « La modernisation de la gestion publique : les leçons de l'expérience » et par quelques articles de bilan qualitatif qui partagent une vision un peu désabusée des effets de stop and go associés aux injonction politiques successives et au manque de suivi de l'administration (Gibert, Thoenig, 1993) (Barouch, 1994). Par la suite les monographies sur la mise en œuvre d'outils de gestion sont moins nombreuses et laissent place à des réflexions plus générales sur les politiques publiques et leur évaluation ou sur la performance publique en lien avec la Loi organique sur les lois de finances (14 articles sur une période deux fois plus longue correspondent en partie à ce modèle de compte rendu). Le seul domaine qui fait exception concerne le contrôle de gestion avec en particulier plusieurs articles consacrés à des tableaux de bord multidimensionnels (balanced scorecard).

Depuis la fin des années 1990, la question des outils est moins mise en avant, cela ne veut pas dire qu'elle est sortie de l'agenda des réformateurs. Les stratégies de réformes ministérielles de 2003 incluent par exemple de nombreux outils de gestion. Et par ailleurs des outils beaucoup moins discutés comme ceux associés à l'informatique et à l'e-administration ne cessent d'être développés.

\section{L'enquête "Changement organisationnel et informatisation»}

L'enquête Changement organisationnel et informatisation a été conçue et coordonnée par le CEE pour la première fois en 1997 (Greenan, HamonCholet, 2000) (Greenan, Mairesse, 2006), en partenariat avec la Dares et le Sessi ${ }^{i}$. Initialement centrée sur les secteurs industriels, elle a en particulier été construite dans le cadre des débats entre économistes sur les effets de l'informatisation dans les entreprises (effet de montée en qualification et «paradoxe de Solow » concernant la faiblesse des gains de productivité au

\footnotetext{
${ }^{4}$ Le ministère de l'éducation nationale et en particulier les universités sont aussi l'objet d'un nombre régulier d'article durant toute la période. En revanche il est remarquable que les ministères de l'économie et des finances ne soient l'objet de quasiment aucun compte rendu.

${ }^{5}$ Dares : direction de l'animation de la recherche, des études et des statistiques, Ministère du travail ; Sessi : Service des études et statistiques du Ministère de l'industrie.
} 
niveau macroéconomique associés à ces nouveaux instruments). L'enquête se compose de deux parties «couplées». D'une part un questionnaire mené auprès de responsables d'entreprises vise principalement à faire un inventaire des outils (gestion et informatique) utilisés par celles-ci. D'autre part un questionnaire auprès de salariés dans ces entreprises permet de faire un état des lieux sur les pratiques organisationnelles et les conditions de travail. La procédure qui consiste à tirer au sort les salariés parmi les entreprises retenues dans l'échantillonnage établi pour l'enquête à destination des employeurs («couplage»), permet de mettre en relation les résultats des deux questionnaires et par exemple de connaître pour un agent donné les outils de gestion présents dans son entreprises (pas nécessairement appliqués à lui) et pour une entreprise développant un type d'outil, les réactions de ses employés.

Pour sa deuxième édition, en 2006 et 2007, l'enquête a été étendue aux autres secteurs marchands, aux hôpitaux et à la fonction publique d'Etat ${ }^{6}$. Cette enquête permet ainsi pour la première fois d'avoir une approche quantifiée et interministérielle sur les pratiques de gestion de l'administration d'Etat $^{7}$ et sur le rôle des outils dans ces évolutions.

De nombreuses enquêtes sociologiques empiriques ${ }^{8}$ conduisent à un certain scepticisme quant aux effets mécaniques d'organisation qui seraient générés par les outils de gestion. Pour ne prendre des exemples que dans le secteur public, Jean Claude Moisdon (1997) et Philippe Bezes (2004) ont mis en évidence des activités de détournement de la finalité première de certains indicateurs. Patrick Le Gales (2004) reconnaît pour sa part une certaine capacité de résistance des "contrôlés». Nils Brunsson (1982) souligne le lien très lâche entre un budget municipal et les dépenses réelles des années qui précèdent et qui suivent. Valérie Boussard (2001) a montré comment parmi des dizaines d'indicateurs de gestion des caisses d'allocations familiale, l'un deux s'était imposé comme la référence unique mobilisée par toutes les composantes de l'organisation alors que de nombreux autres étaient progressivement tombés en désuétude. Ce dernier exemple semble bien pouvoir être généralisé : les outils sont dans tous les cas ressaisis par les acteurs dans le cadre de rapports de force propres à chaque organisation, et de cette ressaisie dépend leur usage effectif ${ }^{9}$.

Prendre acte du caractère situé et interactif de la mobilisation des outils de gestion dans des contextes particuliers, et de leur plasticité, n'interdit

\footnotetext{
${ }^{6}$ Voir note méthodologique en annexe.

${ }^{7}$ II faut cependant citer quelques efforts antérieurs de quantification des pratiques de gestion (Poinsard, 1987) (Rangeon, 1992), (Kuhlman et al., 2008). On peut noter que ces trois articles reposent, comme l'enquête COI, sur un dénombrement de l'usage d'un panier d'outils.

${ }^{8}$ Certains auteurs, souvent parmi ceux qui en dénoncent le plus fermement les effets, accordent a priori un effet aux outils de gestion. Ils convergent ainsi paradoxalement, dans ce déterminisme technologique, avec les cabinets de conseil promoteurs de ces outils (Ogien, 2002).
} 
cependant pas de revenir sur d'éventuels effets propres de certains outils ou sur la réceptivité particulière de certains contextes. Certains outils sont-ils plus facilement mobilisables que d'autres (en particulier, les indicateurs chiffrés simples tendent-ils à supplanter les données qualitatives plus difficiles à mobiliser ?) ou plus malléables que d'autres (les outils informatiques récents organisent-ils le travail ou s'adaptentils au contexte ? (Paucelle, 2007)). En croisant les résultats d'un grand nombre de monographies d'entreprises, Denis Segrestin (2004) a ainsi pu dresser un tableau des effets d'appropriation les plus régulièrement observés dans les entreprises. II distingue ainsi les outils "forts " (par exemple les certifications qualité (Cochoy et al., 1997)) qui forcent les différentes composantes de l'entreprises à prendre parti et des outils "faibles» (par exemple les logiciels de mise en commun des pratiques) qui ne se développent que s'ils trouvent des conditions favorables a priori dans les entreprises. De manière symétrique on peut s'interroger sur la spécificité des contextes de mise en œuvre en termes de secteur d'activité ou de différence public/privé.

L'enquête $\mathrm{COI}$ est mobilisée ici dans cette dernière perspective. En effet, même si la donnée de base est un simple comptage des instruments de gestion déclarés par les directions, la possibilité d'observer la dispersion de la diffusion de ces outils dans les administrations, leurs associations, et surtout, au travers du couplage de l'enquête employeur avec l'enquête employé, les corrélations avec certaines caractéristiques du travail, permettent de s'interroger sur la spécificité du contexte public ou l'impact différencié des outils, sans avoir à présupposer un effet direct de ceux-ci. Nous aborderons en premier lieu le niveau d'équipement en le différenciant par ministère ou regroupements de ministères et discutant le sens des évolutions. En second lieu nous porterons l'attention sur les associations entre différents instruments et les objectifs prioritaires déclarés par les employeurs dans cette enquête, et nous discuterons la pertinence d'une représentation de l'évolution comme modernisation. En troisième lieu nous analyserons les corrélations entre la mobilisation des outils de gestion et les caractéristiques du travail.

\section{La diffusion différenciée des outils dans les ministères}

Le niveau global d'équipement de l'administration d'Etat n'est pas notablement différent de celui des entreprises privées. L'administration est même plutôt plus équipée en outils informatiques que le secteur privé (en moyenne), sauf pour les logiciels informatiques de marché (en opposition avec les applications développées en interne) et certains outils de relation avec les clients. Au sein des outils de gestion, ceux qui sont orientés vers la

\footnotetext{
${ }^{9}$ Cette manière d'apprécier les interactions entre outils de gestion et relations sociales a été développée de longue date dans une perspective historique à propos des outils comptables : à propos de la comptabilité en partie double (Weber, 1991), à propos de la comptabilité analytique (Thomas, Kaplan, 1987) et à propos de l'amortissement (Lemarchand 1993).
} 
qualité de la prestation et la normalisation des prestations aux destinataires sont plus représentés dans le privé que dans l'administration d'État ${ }^{10}$. Cependant, certains outils de gestion, qui étaient l'objet d'expérimentations isolées à la fin des années 1980, comme les tableaux de bord ou les bases de données, sont quasiment généralisés. Même si on ne dispose pas de mesure équivalente à vingt ans de distance, il semble bien que l'on puisse parler d'une relative banalisation de ce qui était exceptionnel alors et, pour ce simple critère comptable, d'une certaine réussite du projet de «modernisation» de la gestion publique.

La diffusion des outils de gestion est très différenciée selon les ministères. Un premier constat s'impose tout d'abord: le plus fort niveau global d'équipement du ministère de l'économie et des finances et le plus faible de la police et du ministère de l'éducation nationale (Tableau I). Sur la liste retenue de 28 outils $^{11}$, les services du ministère de l'économie déclarent en moyenne en utiliser les deux tiers, ceux de l'équipement et de l'agriculture une moitié et ceux de la police ou de l'éduction nationale guère plus d'un tiers. Ce plus faible équipement en outils dans ces dernières organisations est peut-être dû en partie à la petite taille de certaines composantes comme les lycées ou les commissariats : dans le secteur privé la taille de l'entreprise est en effet le premier facteur explicatif des différences d'équipement (Guillemot, Kocoglu, 2010). On peut noter cependant que si on limite aux outils de technologie Internet (délivrance de services sur Internet, possibilité pour l'usager de suivre son dossier en ligne...), le ministère de l'éducation se retrouve en deuxième position, ce qui traduit un usage conséquent de ces instruments tant dans les relations avec le public (élèves, étudiants...) qu'avec les agents (enseignants). La première place du ministère de l'économie n'est véritablement bousculée que pour les outils de gestion des ressources humaines pour lesquels les ministères ingénieurs offrent globalement le plus fort niveau d'équipement.

${ }^{10}$ Pourcentage d'équipement en outils de gestion

\begin{tabular}{|l|l|l|l|}
\hline Outil & Centre d'appel & Norme NF & Norme Iso \\
\hline privé & $47 \%$ & $40 \%$ & $58 \%$ \\
\hline administration & $41 \%$ & $34 \%$ & $37 \%$ \\
\hline \multicolumn{4}{|l}{ Source : Enquête COI-FP, CEE et DGAFP, données pondérées }
\end{tabular}

\footnotetext{
${ }^{11} \mathrm{cf}$. annexe méthodologique pour la liste des outils par catégories. Les données portent sur 298 questionnaires remplis par les directions des services représentant l'administration d'État.
} 
La comparaison avec $2003^{12}$ fait apparaître un mouvement conséquent de croissance globale sur cette période, peut-être lié à des démarches interministérielles comme les stratégies de modernisation dont la mise en œuvre débute après 2003. Le ministère de l'économie, qui avait alors un niveau d'équipement plus élevé que les autres ministères, a maintenu son avance. Ceci peut être mis en relation avec un ensemble de réformes mises en place au début des années 2000 dans ce ministère : démarche diagnostic plan d'action en 2001 à la DGI, débuts d'application du projet Copernic visant à créer un dossier informatique unique (DGI DGCP) pour les contribuables, logiciel Accor de facturation et de paiement intégré (qui n'a cependant pas abouti).

Après avoir dénombré les outils utilisés par les différents ministères, il est intéressant d'observer comment ils sont associés entre eux. Quels outils sont utilisés en même temps que d'autres ou au contraire utilisés de manière antagonique ? L'analyse en correspondances multiples offre une première manière synthétique d'appréhender ces associations d'outils.

Tableau 1 - Taux d'équipement par catégories d'outils

\begin{tabular}{|c|c|c|c|c|c|c|}
\hline \multirow{2}{*}{\multicolumn{2}{|c|}{ Ministère }} & \multicolumn{5}{|c|}{ Outils } \\
\hline & & \multirow{2}{*}{$\begin{array}{c}\text { qualité } \\
26 \%\end{array}$} & \multirow{2}{*}{$\frac{\text { production }}{15 \%}$} & \multirow{2}{*}{$\frac{\text { tic }}{38 \%}$} & \multirow{2}{*}{$\frac{\mathrm{rh}}{63 \%}$} & \multirow{2}{*}{$\frac{\text { total }}{36 \%}$} \\
\hline Éducation & 2007 & & & & & \\
\hline nationale & 2003 & $9 \%$ & $10 \%$ & $24 \%$ & $47 \%$ & $23 \%$ \\
\hline \multirow{2}{*}{$\begin{array}{l}\text { Administration } \\
\text { générale }\end{array}$} & 2007 & $37 \%$ & $13 \%$ & $41 \%$ & $76 \%$ & $42 \%$ \\
\hline & 2003 & $18 \%$ & $9 \%$ & $25 \%$ & $56 \%$ & $27 \%$ \\
\hline \multirow{2}{*}{ Économie } & 2007 & $79 \%$ & $54 \%$ & $56 \%$ & $73 \%$ & $63 \%$ \\
\hline & 2003 & $42 \%$ & $36 \%$ & $32 \%$ & $48 \%$ & $38 \%$ \\
\hline \multirow{2}{*}{$\begin{array}{l}\text { Équipement et } \\
\text { agriculture }\end{array}$} & 2007 & $52 \%$ & $33 \%$ & $41 \%$ & $84 \%$ & $51 \%$ \\
\hline & 2003 & $20 \%$ & $24 \%$ & $20 \%$ & $65 \%$ & $30 \%$ \\
\hline \multirow{2}{*}{ Police } & 2007 & $36 \%$ & $19 \%$ & $23 \%$ & $69 \%$ & $34 \%$ \\
\hline & 2003 & $23 \%$ & $13 \%$ & $16 \%$ & $56 \%$ & $25 \%$ \\
\hline \multirow{2}{*}{ Total } & 2007 & $44 \%$ & $25 \%$ & $42 \%$ & $72 \%$ & $45 \%$ \\
\hline & 2003 & $21 \%$ & $17 \%$ & $25 \%$ & $54 \%$ & $29 \%$ \\
\hline
\end{tabular}

Source : Enquête COI-FP, CEE et DGAFP, données pondérées

\footnotetext{
${ }^{12}$ Pour chacun des outils cités il est demandé si l'outil est présent aujourd'hui (en 2007) et s'il l'était en 2003. La précision de l'information est sûrement plus faible pour les données de 2003 , dans la mesure où elle fait intervenir la mémoire des répondants.
} 


\section{Graphique 1 - Structure d'équipement de la FPE en outils de gestion ; projection des ministères et des objectifs jugés très importants}

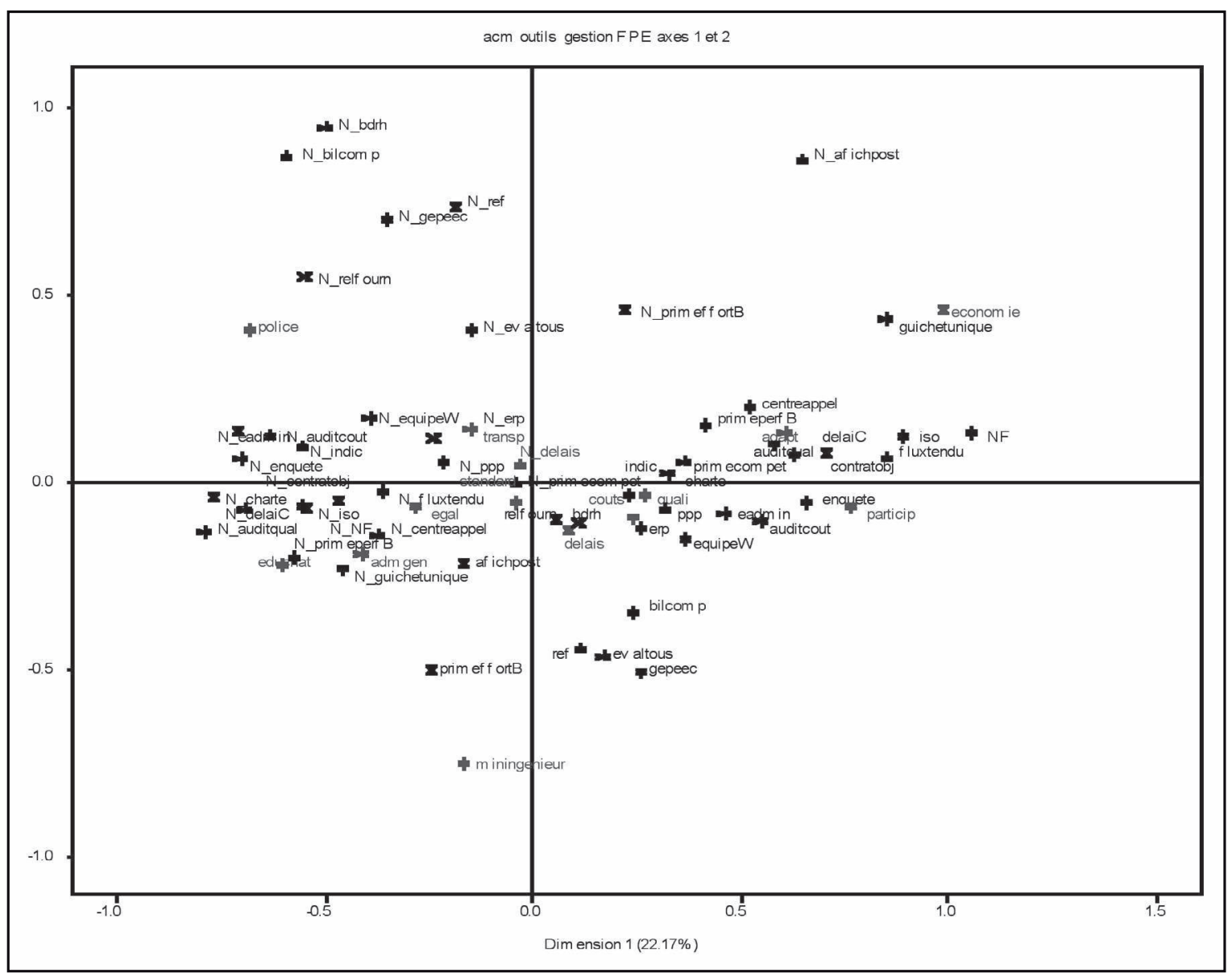

Source : Enquête COI-FP, CEE et DGAFP

Méthodologie : projection des ministères et des objectifs jugés très importants sur le premier plan factoriel de l'analyse en correspondances multiples (ACM) des outils de gestion utilisés par les directions de la FPE (ref = référentiel métier ; bdrh = base de données de ressources humaines ; evaltous = pratique de l'évaluation pour toutes les catégories d'agents ; eadmin = traitement de dossiers individuels en ligne ; enquête = enquête de qualité menées auprès des usagers ; bilcomp = bilan de compétences ). 


\section{Les outils appellent les outils}

Tout d'abord un mécanisme d'accumulation des outils s'impose de manière très nette par l'inertie forte du premier axe horizontal $(22 \%)$ et par le fait qu'il oppose clairement la disposition des outils de gestion dans les directions à leur absence ${ }^{13}$. II y a en quelque sorte un parcours progressif d'accès aux outils de gestion, les services acquérant les outils les plus sophistiqués (reportés le plus à droite sur le graphique I) sans se débarrasser des outils plus communs. Cet effet de cumul n'a rien de spécifique au public; on le retrouve dans l'enquête sur le secteur privé (Guillemot, Kocoglu, 2010). Parmi les outils les plus «en pointe», qui viennent s'ajouter aux autres, on trouve les normes de qualité (NF et ISO), c'est-à-dire les «outils forts" décrits par Denis Segrestin, dont l'impact sur l'organisation pourrait être particulièrement contraignant.

On vérifie que «les outils appellent les outils» quelle que soit leur catégorie en observant la corrélation entre des indicateurs de synthèse d'équipement, construits dans les trois principales fonctions que nous avons retenu, les outils $\mathrm{RH}$, de gestion de la production, de suivi de la qualité, et les $\mathrm{TIC}^{14}$. Toutes les corrélations sont positives et fortes. Cependant, la corrélation entre les outils de ressources humaines et les outils de la qualité, toujours positive, est un peu plus faible.

Tableau 2 - Corrélations entre types d'outils

\begin{tabular}{|l|l|l|l|l|}
\hline & $\begin{array}{l}\text { Indicateur } \\
\text { de synthèse } \\
\text { outils RH }\end{array}$ & $\begin{array}{l}\text { Indicateur de } \\
\text { synthèse outils } \\
\text { de production }\end{array}$ & $\begin{array}{l}\text { Indicateur de } \\
\text { synthèse outils } \\
\text { gestion de la qualité }\end{array}$ & $\begin{array}{l}\text { Indicateurs } \\
\text { de synthèse } \\
\text { outils TIC }\end{array}$ \\
\hline $\begin{array}{l}\text { Indicateur de synthèse } \\
\text { outils RH }\end{array}$ & 1,00 & $0,19 * * *$ & $\mathbf{0 , 1 3 * *}$ & $0,36^{* * * *}$ \\
\hline $\begin{array}{l}\text { Indicateur de synthèse } \\
\text { outils de production }\end{array}$ & 1,00 & $0,28 * * *$ & $0,34 * * *$ \\
\hline $\begin{array}{l}\text { Indicateur de synthèse } \\
\text { outils gestion de la } \\
\text { qualité }\end{array}$ & & & 1,00 & $0,34 * * *$ \\
\hline $\begin{array}{l}\text { Indicateurs de } \\
\text { synthèse outils TIC }\end{array}$ & & & & 1,00 \\
\hline
\end{tabular}

Source : Enquête COI-FP CEE et DGAFP, $N=298$ Coefficients de corrélation partielle de Spearman, probabilité ${ }^{*}<0,1,{ }^{* *}<0,05^{* * *}<0,01$

\footnotetext{
${ }^{13} \mathrm{~A}$ l'exception de deux outils de ressources humaines, nous y reviendrons.

${ }^{14} \mathrm{Ces}$ indicateurs synthétiques d'intensité d'équipement correspondent à un comptage simple du nombre d'outils de la fonction considérée utilisés par la direction. Pour la liste d'outils par fonction, voir l'encadré méthodologique.

La corrélation entre ces indicateurs est établie en contrôlant les ministères. La corrélation, pour une échelle d'intensité d'équipement porte sur l'ordre (coef de spearman).
} 


\begin{abstract}
Une modernisation?
Si ce mode de diffusion des outils de gestion est aussi observé dans le secteur privé, une interprétation propre au secteur public peut cependant être engagée lorsqu'on les associe aux réponses sur l'importance des objectifs d'amélioration, ou des moyens utilisés, dans quatorze domaines proposés dans l'enquête, qui vont de la qualité ou de la réduction des coûts, à la participation des usagers ${ }^{15}$. Ces objectifs et moyens correspondent en partie à un débat ouvert par le rapport Silicani (2008) sur les «valeurs» de la modernisation. On remarque en effet une distribution des termes associés à ces «valeurs » sur le premier axe, l'égalité et la transparence, des valeurs associées au droit, étant positionnées à proximité de l'absence d'utilisation des outils de gestion, alors que ce que l'on peut considérer comme des valeurs associées à l'efficacité se trouve projeté du côté de leur utilisation. On peut distinguer parmi ces dernières les valeurs «modernes» liées à la recherche d'efficacité globale (la baisse des coûts, la qualité, la réduction des délais), des valeurs «post modernes» comme la différenciation des destinataires et à leur participation à la décision. Ces dernières apparaissent proches de l'utilisation des outils les plus en pointe alors que les précédentes correspondent au main stream de la transformation gestionnaire. On vérifie avec les indicateurs de synthèse que, alors que les indicateurs des valeurs « nouvelles » sont largement associés aux outils de gestion, les corrélations entre la mise en avant de la finalité d'égalité et les outils de gestion sont non significatives, et même légèrement négative entre la quête de la transparence ${ }^{16}$ et le développement des outils les plus sophistiqués de la gestion de la production.
\end{abstract}

\footnotetext{
${ }^{15}$ Les questions traitées ici sont libellées de la manière suivante : " quelle est aujourd'hui l'importance des objectifs d'amélioration (versus des moyens) suivants ? " avec une liste de 5 objectifs d'amélioration, et de 9 moyens, et une gradation à quatre niveau (de nulle à très forte). La réponse « forte " est largement dominante pour l'ensemble des items, la différenciation étant plus importante sur la modalité " très forte ". C'est la modalité « très forte » qui a été reportée dans l'ACM, tandis que pour l'analyse des corrélations, c'est une échelle de l'ensemble des quatre réponses qui est utilisée.

${ }^{16}$ Jean Ludovic Silicani présente pourtant la transparence comme une valeur nouvelle. Elle arrive en troisième position dans un sondage ifop de janvier 2008 sur les valeurs promues par les fonctionnaires après l'efficacité et la qualité.
} 
Tableau 3 - Corrélations entre utilisation d'outils et «valeurs»

\begin{tabular}{|l|l|l|l|l|}
\hline \multicolumn{1}{|c|}{ Usage des outils } & \multicolumn{1}{c|}{ RH } & Production & \multicolumn{1}{c|}{ Qualité } & TIC \\
\hline Importance de la qualité & $0,14 * *$ & $0,12 * *$ & $0,27 * * *$ & $0,15 * * *$ \\
\hline Importance des coûts & $0,18 * * *$ & $0,12 * *$ & $0,10 *$ & $0,20 * * *$ \\
\hline Importance de la standardisation & $\mathrm{ns}$ & $0,13 * *$ & $0,12 * *$ & $\mathrm{~ns}$ \\
\hline Importance de la transparence & $\mathrm{ns}$ & $\mathbf{- 0 , 0 9 *}$ & $\mathrm{ns}$ & $\mathrm{ns}$ \\
\hline Importance de l'égalité & $\mathbf{0 , 1 1 *}$ & $\mathrm{ns}$ & $\mathrm{ns}$ & $\mathrm{ns}$ \\
\hline $\begin{array}{l}\text { Importance de la motivation du } \\
\text { personnel }\end{array}$ & $\mathbf{0 , 1 4 * *}$ & $\mathrm{ns}$ & $\mathrm{ns}$ & $\mathrm{ns}$ \\
\hline $\begin{array}{l}\text { Importance de la participation des } \\
\text { usagers }\end{array}$ & $0,23 * * *$ & $0,19 * * *$ & $0,09 *$ & $0,22 * * *$ \\
\hline $\begin{array}{l}\text { Importance de l'adaptation à } \\
\text { différents publics }\end{array}$ & $0,17 * * *$ & $\mathrm{~ns}$ & $0,23 * * *$ & $0,14 * *$ \\
\hline
\end{tabular}

Source : Enquête COI-FP CEE et DGAFP, $N=298$ Coefficients de corrélation partielle de Spearman, probabilité ${ }^{*}<0,1,{ }^{* *}<0,05{ }^{* * *}<0,01$

\section{La position singulière des outils de ressources humaines}

Si ce premier facteur de différenciation lié au degré d'équipement (tous outils confondus) semble ainsi en première analyse soutenir la représentation du changement comme une "modernisation" unidimensionnelle, la lecture du second axe de l'ACM invite cependant à creuser une possible ligne de différenciation dans cette représentation en portant l'attention sur les outils de ressources humaines. Le second facteur de différenciation conduit en effet à opposer les services qui n'utilisent pas d'outils de ressources humaines à ceux qui en utilisent. Cette spécificité des outils de ressources humaines dans la panoplie des outils de gestion avait déjà été repérée précédemment: c'est la seule catégorie pour laquelle le ministère de l'économie n'est pas le plus équipé et c'est celle dont le niveau de corrélation avec les autres outils (en particulier ceux de la qualité) est le plus faible.

La lecture du second axe de l'ACM conduit aussi à spécifier l'approche des outils de ressources humaines. On remarque en effet en premier lieu que les différents types de primes ne sont pas positionnés de la même manière : si l'usage d'une " prime liée à l'effort, à la manière de servir » est associée à l'utilisation d'outils de ressources humaines, la mobilisation d'une « prime liée aux résultats » est plutôt associée à l'usage des autres catégories d'outils de gestion.

Les impacts sur le travail
Le couplage des enquêtes auprès des employeurs et des employés permet de relier les données sur la diffusion des outils de gestion avec des informations sur le travail. 
L'enquête sur le travail ${ }^{17}$ permet alors d'aborder deux questions qui prennent un sens particulier dans le débat sur la bureaucratie : la question du contrôle et celle de la différenciation. La critique de la bureaucratie portée par le courant du management public a en effet mis l'accent sur la déresponsabilisation des cadres, qui n'ont les moyens ni de réellement contrôler un travail routinier ni d'inciter à l'engagement des agents par des récompenses pécuniaire immédiates (prime) ou différées (avancement plus rapide dans la carrière).

\section{Les différences entre ministères}

Un certain nombre de caractéristiques du travail se différencient très clairement selon les ministères, les différences entre ministères étant d'ailleurs, de manière remarquable, supérieures à celle que l'on peut observer entre les différents secteurs d'activités marchandes (tableau 4). Les causes de ces différences peuvent être diverses. L'une d'elle est sûrement la différence des prestations accomplies par les ministères : fonction d'ordre dans l'espace public pour la police, traitement de masse de dossier pour les finances, mise en œuvre de politiques publiques territorialisées pour les ministères ingénieurs.

Tableau 4 - Déclaration des salariés sur le contrôle du travail

\begin{tabular}{|l|l|l|l|l|l|l|l|l|l|}
\hline $\begin{array}{l}\text { Proportion de } \\
\text { salariés qui } \\
\text { déclarent : }\end{array}$ & $\begin{array}{l}\text { Education } \\
\text { nationale }\end{array}$ & $\begin{array}{l}\text { Administration } \\
\text { générale }\end{array}$ & $\begin{array}{l}\text { Economi } \\
\text { finances }\end{array}$ & $\begin{array}{l}\text { Equipment } \\
\text { Agriculture }\end{array}$ & Police & $\begin{array}{l}\text { Total } \\
\text { FPE }\end{array}$ & $\begin{array}{l}\text { Total } \\
\text { privé }\end{array}$ & $\begin{array}{l}\text { Activités } \\
\text { financières }\end{array}$ & $\begin{array}{l}\text { Services } \\
\text { aux } \\
\text { entreprises }\end{array}$ \\
\hline $\begin{array}{l}\text { Avoir des } \\
\text { salariés sous } \\
\text { (leur) ordre } \\
\text { ou autorité }\end{array}$ & 29 & 23 & 25 & 28 & 34 & 28 & 30 & 24 & 29 \\
\hline $\begin{array}{l}\text { Travail } \\
\text { contrôlé au } \\
\text { moins une } \\
\text { fois par mois }\end{array}$ & 43 & 45 & 62 & 61 & 69 & 55 & 61 & 59 & 60 \\
\hline $\begin{array}{l}\text { Surveillance } \\
\text { informatique }\end{array}$ & 8 & 20 & 50 & 22 & 38 & 26 & 26 & 31 & 26 \\
\hline $\begin{array}{l}\text { Application } \\
\text { stricte des } \\
\text { ordres et } \\
\text { procédures }\end{array}$ & 56 & 59 & 65 & 53 & 65 & 60 & 54 & 50 & 55 \\
\hline $\begin{array}{l}\text { Impossibilité } \\
\text { fréquente de } \\
\text { respecter à la } \\
\text { fois qualité et } \\
\text { délais }\end{array}$ & 9 & 11 & 12 & 7 & 12 & 10 & 10 & 14 & 9 \\
\hline
\end{tabular}

Source : Enquête COI-FP, CEE et DGAFP (valeurs maximales en gras), données pondérées

${ }^{17}$ Les traitements portent sur environ 1000 questionnaires salariés. 
Les niveaux de contrôle déclarés par les agents de l'Etat sont plus élevés lorsqu'ils travaillent dans la police et au ministère de l'économie, ils dépassent même ceux déclarés par les salariés du secteur privé. Cependant les formes de ce contrôle apparaissent d'emblée différentes. Dans le cas de la police, cela se traduit tout particulièrement par une hiérarchie plus finement découpée que dans le reste de l'administration et que dans le privé (proportion plus élevée d'agents déclarant avoir la responsabilité hiérarchique sur d'autres). Cette forme de contrôle hiérarchique est par ailleurs associée au plus faible niveau d'équipement en outils de gestion.

Les agents du ministère de l'économie sont un peu moins nombreux à déclarer un contrôle fréquent du travail, mais ils se caractérisent par un niveau élevé de surveillance du travail par l'informatique, que l'on peut considérer comme un indicateur du contrôle indirect par les instrumentations informatiques et gestionnaires. L'importance de cette dimension du contrôle par les instruments dans ce ministère se trouve confirmée par les réponses aux questions destinées à capter l'effet de démarches de qualité ou de normalisation du travail. La proportion d'agents qui déclarent leur travail soumis à une définition précise des objectifs ou des procédures est en effet nettement supérieure au reste de l'administration et dépasse même parfois celles observées dans le secteur privé.

Les indicateurs de pratiques d'incitation financière ou de différenciation des carrières des agents ${ }^{18}$ révèlent en premier lieu une différence nette avec le privé (tableau 5). Dans tous les regroupements de ministères considérés, et pour toutes les questions les niveaux de perception de la différenciation des agents sont nettement plus faibles que dans le secteur privé, ce qui confirme des résultats récurrents (Rainey, Bozeman, 2000). En second lieu les ministères de l'équipement et de l'agriculture présentent un fort niveau relatif pour l'ensemble des questions ce qui établit un lien entre la mesure de l'outillage en ressources humaines et la perception par les agents de pratiques dérogeant au modèle canonique de l'ancienneté. Enfin on peut remarquer, en troisième lieu, le cas de figure singulier du ministère de l'économie, avec le décalage entre un sentiment relativement fort des agents que leur salaire ou avancement dépend de leur travail et le faible nombre des "chefs» qui déclarent avoir une influence sur les salaires et la promotion des personnes qu'ils encadrent. Ce décalage pourrait traduire un faible niveau de délégation des politiques d'incitation.

\footnotetext{
${ }^{18}$ Les indicateurs retenus correspondent aux réponses positives aux questions suivantes : Une partie de votre rémunération est-elle variable ? L'augmentation de votre rémunération ou l'avancement dépend-elle du travail effectué ? Avez-vous de l'influence sur les primes ou la promotion d'autres agents ; L'entretien d'évaluation porte-t-il sur des critères précis et/ou mesurables?
} 
Tableau 5 - Incitations et différenciation des salariés

\begin{tabular}{|l|l|l|l|l|l|l|l|l|l|}
\hline & $\begin{array}{l}\text { Education } \\
\text { nationale }\end{array}$ & $\begin{array}{l}\text { Administration } \\
\text { générale }\end{array}$ & $\begin{array}{l}\text { Economie } \\
\text { finances }\end{array}$ & $\begin{array}{l}\text { Equipment } \\
\text { Agriculture }\end{array}$ & Police & $\begin{array}{l}\text { Total } \\
\text { FPE }\end{array}$ & $\begin{array}{l}\text { Total } \\
\text { privé }\end{array}$ & $\begin{array}{l}\text { Activités } \\
\text { financières }\end{array}$ & $\begin{array}{l}\text { Services } \\
\text { aux } \\
\text { entreprises }\end{array}$ \\
\hline $\begin{array}{l}\text { salaire } \\
\text { variable }\end{array}$ & 13 & 22 & 22 & $\mathbf{2 9}$ & 20 & 20 & 41 & 40 & 34 \\
\hline $\begin{array}{l}\text { salaire ou } \\
\text { avancement } \\
\text { dépend } \\
\text { du travail } \\
\text { individuel ou } \\
\text { collectif }\end{array}$ & 17 & 28 & $\mathbf{3 7}$ & $\mathbf{3 5}$ & 19 & 27 & 59 & 58 & 56 \\
\hline $\begin{array}{l}\text { influence sur } \\
\text { les salaires et } \\
\text { la promotion } \\
\text { des personnes } \\
\text { encadrées }\end{array}$ & 14 & $\mathbf{4 6}$ & 22 & $\mathbf{4 1}$ & 15 & 24 & 54 & 51 & 55 \\
\hline $\begin{array}{l}\text { évaluation sur } \\
\text { critères précis }\end{array}$ & 84 & 81 & 89 & $\mathbf{9 5}$ & 93 & 88 & 92 & 96 & 90 \\
\hline
\end{tabular}

Source : Enquête COI-FP, CEE et DGAFP, données pondérées

\section{Les outils de gestion ont-ils un impact sur le travail, «toutes choses égales par ailleurs »?}

Lorsqu'on met en relation les réponses données par les agents interrogés et les outils déclarés par leurs employeurs «toutes choses égales par ailleurs» c'est-à-dire en contrôlant l'effet de l'appartenance à un ministère, de la catégorie socioprofessionnelle, du statut (fonctionnaire/contractuel), de la localisation (administration centrale/services déconcentrés) et d'un travail en relation directe avec le public, les corrélations significatives sont rares ${ }^{19}$. $\mathrm{Ce}$ résultat rejoint ce que l'on observe dans le secteur privé ${ }^{20}$. Dans ce contexte, il est alors intéressant de s'intéresser aux outils qui se détachent, pour lesquels des corrélations significatives apparaissent avec les réponses des salariés. Deux tendances se dégagent ainsi (tableau 6) ${ }^{21}$.

\footnotetext{
${ }^{19}$ Les indicateurs de synthèse utilisés précédemment ne sont ainsi corrélés de manière significative à $5 \%$ à aucun des items examinés dans le questionnaire employé. II faut donc regarder plus finement les outils précis qui peuvent être corrélés avec certaines caractéristiques du travail.

${ }^{20}$ Deux raisons peuvent être avancées. La première est liée à la méthode, les salariés interrogés, tirés au sort dans le service ne sont pas toujours concernés par un outil particulier (certains, comme le personnel d'entretien des locaux peut ne l'être par quasiment aucun), d'où un effet de dilution de l'effet mesuré des outils. En second lieu, les effets sur le travail peuvent naître de la conjonction d'outils plutôt que d'outils pris séparément.

${ }^{21}$ Dans un objectif de simplification, nous n'avons retenu, dans le tableau 6 , que les outils et les questions aux salariés pour lesquels les corrélations significatives les plus nombreuses apparaissaient. (suite p. 90)
} 


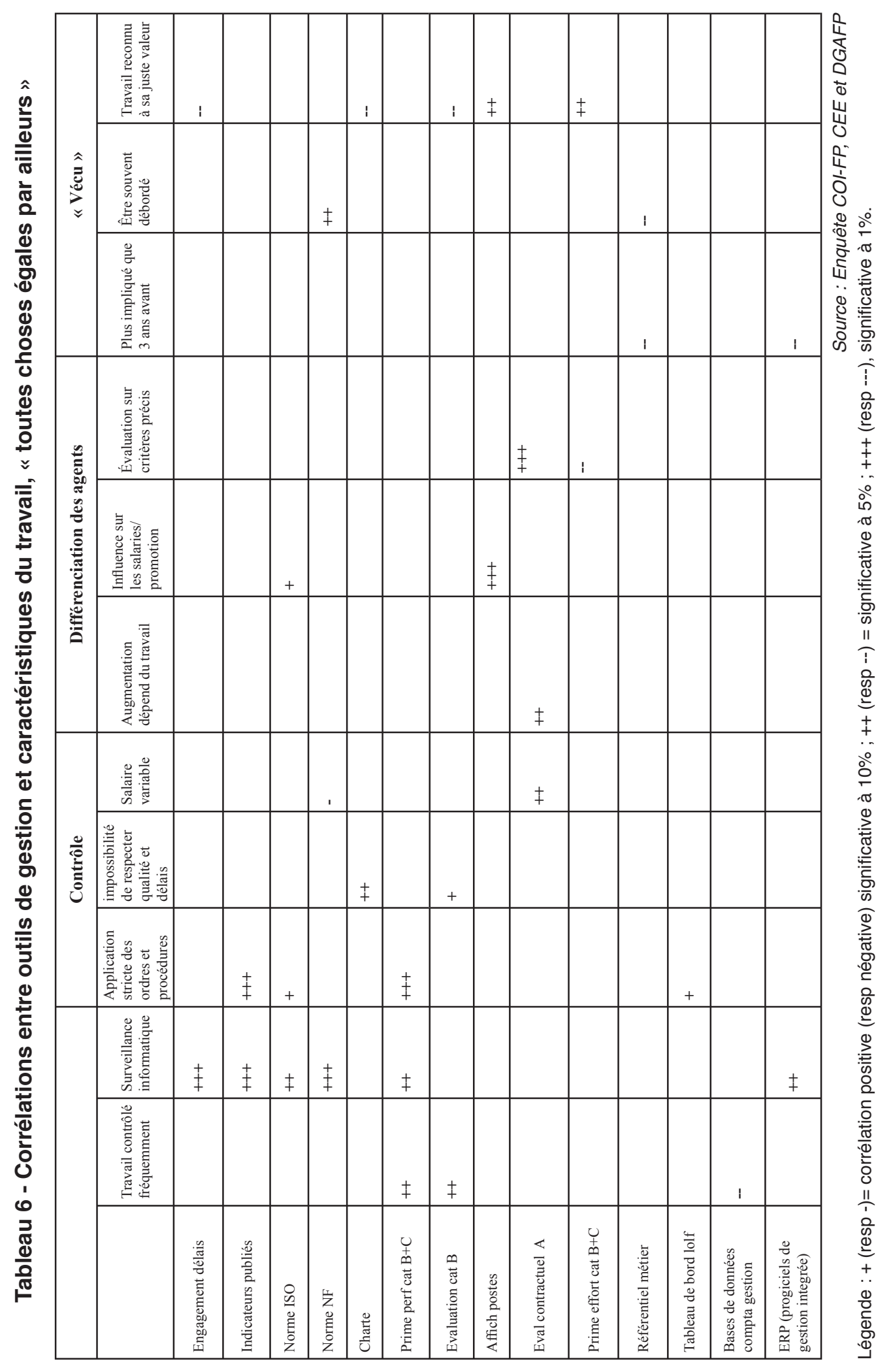


Tout d'abord, les outils qui comprennent explicitement des engagements précis sur la procédure et les résultats (publication d'indicateurs, normes de qualité, primes à la performance) sont associés à des effets sur le travail conformes à leur théorie : plus de contrôle, en particulier de manière indirecte par l'ordinateur et plus de procédures appliquées à la lettre. On retrouve l'argument des «outils forts» développé par Denis Segrestin (2004). L'expression d'une impossibilité de concilier qualité et délais que l'on trouve associée à l'existence de chartes et à l'évaluation des agents de catégorie B apparaît cependant aussi avec d'autres outils directement liés au traitement de masse des usagers, ce qui peut à ce titre être associé autant à la situation de contact continu avec le public ${ }^{22}$ qu'à l'usage d'un instrument particulier.

Ces outils correspondent à une conception techniciste de la qualité largement centrée sur un contrôle des procédures ou des outputs et qui conduisent à un renforcement de la prescription. Cette conception de la qualité s'oppose à une autre approche qui repose au contraire sur un certain relâchement des contraintes exercées sur les agents leur permettant de s'adapter à des situations singulières. Cette seconde approche qui joue sur la capacité d'adaptation et d'invention au cœur du travail a été mise en évidence dans de nombreuses enquêtes qualitatives (Jeannot, 2008b) et dans quelques enquêtes quantitatives. Dans une enquête menée sur 500 «petits fonctionnaires » de I'Isère, Philippe Warin (2002) montre que plus d'un tiers des agents déclarent adapter les règles et procédures et ceci très largement pour retrouver le sens réel de la règle ou de la prestation de service. Les enquêtes statistiques sur les conditions de travail menées tous les sept ans par la Dares depuis la fin des années soixante-dix indiquent aussi une légère montée des indicateurs de l'autonomie au travail entre 1991 et 1998 suivie d'un tassement entre 1998 et $2005^{23}$.

\footnotetext{
${ }^{22}$ Les corrélations sont faites en contrôlant par la réponse « travaille en relation avec le public » mais la réponse positive à cette question mêle les contacts fréquents et rares avec le public.

${ }^{23}$ Evolution d'indicateurs d'autonomie de salariés des entreprises et des trois fonctions publiques :
}

\begin{tabular}{|l|c|c|c|c|c|c|}
\hline \multirow{2}{*}{ Les salariés déclarent : } & \multicolumn{2}{|c|}{1991} & \multicolumn{2}{c|}{1998} & \multicolumn{2}{c|}{2005} \\
\cline { 2 - 7 } & entreprises & 3 FP & entreprises & 3 FP & entreprises & 3 FP \\
\hline Ne suivre ni ordres ni consignes & 17,2 & 18,2 & 19,7 & 20,2 & 21,3 & 19,5 \\
\hline La hiérarchie indique les objectifs & 78,7 & 83,5 & 84,2 & 87,3 & 79,8 & 84,3 \\
\hline
\end{tabular}

Source : Enquêtes Conditions de travail, DARES

${ }^{24}$ Les catégories $B$ et $C$ ont été retenues pour cet indicateur (primes à la performance) plutôt que la catégorie A, car c'est sur ces catégories que l'on observe des corrélations. 
La seconde tendance concerne les outils de ressources humaines. Les corrélations avec des caractéristiques particulières de travail sont plus nombreuses que pour les autres catégories d'outils. Cependant, les effets des outils $\mathrm{RH}$ ne se laissent pas réduire à un argument simple. La pratique d'une prime de performance pour les catégories B et $C^{24}$ est associée plutôt aux indicateurs du renforcement du contrôle qu'à ceux liés à la différenciation des agents. La pratique de l'évaluation pour les fonctionnaires de catégorie $B$ est de même associée à plus de contrôle du travail. Ceci confirme le lien, déjà noté dans l'analyse de l'équipement des ministères, de ces pratiques avec les instruments de contrôle de la qualité et d'engagement précis. La «prime de performance » apparaît ainsi plutôt comme un complément de la rationalisation de la production dans une lignée toute taylorienne. On remarque également les corrélations établies entre un certain nombre de caractéristiques du travail et trois indicateurs : l'attribution de primes liées "à l'effort à la manière de servir» pour les catégories $B$, la pratique de l'évaluation pour les contractuels de catégorie $A^{25}$ et l'affichage de postes. Ces indicateurs sont associés à des pratiques de différenciation. On peut alors considérer qu'il s'agit d'outils ayant une influence directe sur l'évolution des relations humaines au sein de l'administration.

Parmi les indicateurs qui se dégagent dans cette seconde tendance, certains ne peuvent être tout à fait considérés comme des «outils". Ainsi, il n'existe pas de dispositif précis correspondant à une "prime liée à l'effort, la manière de servir ». Une réponse positive doit donc être plutôt considérée comme la marque d'une attitude vis-à-vis du personnel. II en va de même pour "l'évaluation des agents contractuels". Alors que les entretiens d'évaluation, rendus obligatoires depuis quelques années, avec des routines de formulaires à faire remonter, sont quasiment généralisés à l'ensemble des fonctionnaires, à peine la moitié des responsables de service déclarent évaluer les contractuels. Cette démarche d'évaluation en direction des non fonctionnaires, au moment de l'enquête, dépend de la volonté du chef de service. II est possible ainsi de lire cet indicateur comme la marque d'un certain volontarisme dans la pratique de l'évaluation ${ }^{26}$. Ce que capte ici l'enquête est donc moins l'effet de la seule présence d'un outil, que certaines indications sur une attitude des responsables de service qui prennent au sérieux leur usage ${ }^{27}$.

\footnotetext{
${ }^{25}$ Des effets similaires mais moins marqués sont observables pour les primes à l'effort pour les catégories $A$ et l'évaluation des contractuels $B$.

${ }^{26}$ On vérifie que les écarts entre les réponses des agents dans les directions qui déclarent évaluer les contractuels et les autres ne sont pas dues aux réponses des contractuels eux-mêmes (qui seraient plus nombreux dans ces directions, et auraient des réponses différentes de celles des fonctionnaires) en contrôlant la corrélation par le statut des agents.

${ }^{27}$ L'enquête auprès des salariés permet aussi d'appréhender la pratique de la formation. Les corrélations établies entre le fait d'avoir suivi une formation et les indicateurs d'évolution du travail confirment la seconde tendance : corrélation positive avec les augmentations qui dépendent du travail, des cadres qui pensent influencer la promotion de leurs subordonnés, une évaluation sur critère précis, une implication plus forte, mais aussi la surveillance par informatique.
} 
Ces deux tendances se retrouvent aussi lorsque l'on s'intéresse au « vécu » des agents à travers une question sur la fréquence du sentiment d'être débordé (indicateur de stress), une question portant sur l'évolution positive ou négative, depuis 3 ans, de l'implication dans le travail et enfin une question portant sur le sentiment de reconnaissance de la valeur du travail. II ressort que les outils de qualité sont plutôt associés à plus de stress et à un sentiment d'absence de reconnaissance de la valeur du travail, alors que l'affichage des postes ou la prime liée à l'effort sont associés à un plus de reconnaissance de la valeur du travail. De son côté, l'existence d'un référentiel métier semble associé à la caricature traditionnelle des fonctionnaires : moins d'implication et peu de stress.

Une dernière remarque concerne, en creux, les outils directement associés à la mesure de la performance censés permettre à la direction d'avoir un accès sans médiation aux données des différentes composantes de l'administration : les tableaux de bord, les bases de données de comptabilité et gestion, très largement diffusées, ou la version informatique moderne du suivi intégré de la gestion (ERP, Enterprise ressource planning). Ces outils particuliers sont considérés par beaucoup ${ }^{28}$ comme cruciaux pour une nouvelle gestion publique. Cependant, ils ne sont associés directement qu'à peu de caractéristiques du travail, telles qu'elles peuvent être mesurées dans l'enquête, voire parfois à des caractéristiques particulières qui vont dans le sens opposé à celui d'une plus grande maîtrise sur le travail accompli. Ainsi le modèle de contrôle des coûts inspiré de " bonnes pratiques " d'administrations étrangères semble moins marquer l'activité quotidienne des fonctionnaires d'Etat que le transfert de techniques de la qualité issues plutôt de l'expérience des entreprises publiques françaises ${ }^{29}$.

On retrouve ainsi au niveau de l'analyse des corrélations simples entre les outils dont disposent les employeurs et les réponses du questionnaire auprès des employés une partition comparable à celle notée dans l'analyse de l'association des outils entre eux. D'un coté, des outils orientés vers la rationalisation de la production, qui incluent la prime de performance, sont associés à des caractéristiques mesurables de mise au travail des agents par un contrôle accru, sans que les pratiques de différenciation de ceux-ci ne soient véritablement renforcées. De l'autre côté, les outils de ressources humaines et, au-delà même des outils, une certaine manière de prendre au sérieux la "question des hommes", associés soit à une différenciation perçue par les agents et par les cadres, soit à une meilleure perception par les agents de leur gestion. Dans les deux cas les rares "outils» pour lesquels des corrélations significatives sont notées avec le questionnaire auprès des employés peuvent être considérés comme la «pointe avancée »

\footnotetext{
${ }^{28}$ En témoigne la place tenue par les articles sur ce type d'outils dans la revue politiques et management public.

${ }^{29}$ Cet argument de l'influence du modèle des entreprises publiques françaises sur l'administration de l'Etat a été développé pour ce qui concerne la réforme de la fonction publique dans (Jeannot, 2008a).
} 
de l'outillage de gestion. Ils viennent en effet en plus des autres (surtout pour les outils de la qualité (NF ISO)), si bien que l'effet observé peut être en partie lié au cumul et non au seul dernier instrument ajouté. Ces pointes avancées de l'outillage et des pratiques permettent cependant d'affiner la première ligne de partage. Du côté des outils de la rationalisation, ce sont bien ceux qui se situent en aval d'engagements précis sur des prestations plus que les outils amont de pilotage des politiques (par exemple les audits coûts) qui font la différence, alors qu'avec la mise en œuvre de la LOLF (l'enquête s'est déroulé la première année de sa mise en place généralisée) ce sont les seconds qui sont présentés comme les outils les plus symboliques de la réforme. Du côté des ressources humaines, c'est moins l'accumulation des outils que l'attitude des responsables de services qui semble associée à des effets de différenciation.

\section{Conclusion}

\section{Une technicisation pour une réforme discrète}

La dimension gestionnaire et plus particulièrement la diffusion d'outils normalisés de gestion demeure une composante centrale de la pratique de réforme de l'administration d'Etat. On peut être tenté d'y voir une victoire après coup des projets des années 1990. Cette conception est soutenue par le maintien d'une spécificité de l'orientation ressources humaines dans les ministères ingénieurs qui avaient été à la pointe durant cette période. Mais la nette progression de l'équipement entre 2003 et 2007, l'émergence d'outils nouveaux, et la coexistence possible d'une réforme orientée qualité avec un certain "conservatisme " concernant la différenciation des agents, montre que l'on est loin de vivre les simples effets retardés des politiques explicites de «modernisation» par les outils de gestion de la fin des années 1980. On peut à l'inverse lire dans cette dimension technique de la réforme une conséquence de l'échec d'autres modalités de réforme, comme dans le cas du développement des technologies Internet de pointe au ministère de l'éducation nationale dans un contexte de faible transformation gestionnaire de ce ministère ou lorsque les ministères de l'économie et des finances lancent une opération de fusion des bases de données et outils informatiques suite à un échec de la fusion des services.

De manière plus générale, il semble que l'on puisse lire dans ce développement maintenu des outils de gestion une forme discrète de réforme. La réorganisation effective qui intervient de fait quelques années après le rapprochement informatique des fichiers au ministère des finances peut alors être mise au crédit de cette interprétation. La diffusion d'outils informatiques non controversés (micro informatique, Internet, ..) participe aussi de cet alignement sur le secteur privé. Si on peut considérer que l'administration est plongée (éventuellement avec un léger décalage) dans les évolutions générales de la production, on rejoint aussi l'analyse de Boukaert (2003) sur la réforme dans les pays continentaux qui serait finalement moins difficilement menée par cette voie de modernisation des outils de gestion dans des contextes où la remise en cause frontale des principes de l'administration trouve trop peu d'écho. 


\section{Deux modèles de réforme}

Cette voie de réforme par les outils n'est pas unitaire. Les différentes composantes de l'enquête $\mathrm{COI}$ permettent de faire émerger une double attitude possible de réforme, d'un côté une réforme par les outils de gestion rationalisant la production et tout particulièrement les outils impliquant des engagements de qualité de service, de l'autre une réforme qui repose sur un renouveau des relations de l'administration avec ses fonctionnaires.

Les outils organisant la production ont connu une phase d'expansion ces dernières années. D'une manière générale, la diffusion des outils de gestion s'inscrit dans une dynamique globale, dans laquelle ils viennent se compléter les uns les autres dans un ordre hiérarchisé d'acquisition de méthodes de plus en plus contraignantes. Cette dynamique est directement associée au relais, par les responsables des services, des valeurs de la modernité (diminution des coûts et délais) ou d'une post modernité (participation des usagers, adaptation) pour les plus sophistiqués, en opposition aux valeurs de transparence et d'égalité plus liées à l'absence d'équipement. Parmi ces outils, ceux qui incluent une dimension d'engagement de qualité de la prestation (norme ISO et $\mathrm{NF}$, engagement de délais, charte ...) sont directement corrélés à des tendances à la mise sous contrôle du travail et souvent à des signaux négatifs émis par les agents (stress, sentiment de non reconnaissance de la valeur du travail). En revanche, la méthode retenue ne permet pas de faire émerger une corrélation nette entre ces outils et les actions de différenciation des carrières des agents. Des pratiques conformes au nouveau modèle de la gestion publique peuvent ainsi être associées à des aspects décrits comme archaïques par les mêmes doctrines. On retrouve ici la dimension fondamentalement hybride de la réforme administrative décrite par Emery et Giauque (2007).

Les outils associés aux ressources humaines occupent une position singulière, ici aussi appréhendée à travers plusieurs angles. Ils sont moins fortement associés aux autres outils et ce sont les seuls pour lesquels le ministère de l'économie et des finances n'est pas le plus équipé. Lorsque l'on porte l'attention sur les perceptions des salariés, les différences les plus marquées semblent davantage associées à des indicateurs indirects d'attitude vis-à-vis des ressources humaines qu'à la simple possession d'un outil. La perception par les agents d'une différenciation de traitement est, en particulier, associée à l'engagement plus fort de l'encadrement dans l'évaluation. Le sentiment de reconnaissance du travail parait lié à l'affirmation par la direction d'une volonté de reconnaître l'effort, la manière de servir, ou de faciliter les évolutions de carrières, et non à un dispositif gestionnaire précis.

Cette opposition entre deux modalités de la réforme, qui se dégage aussi indépendamment du ministère d'appartenance, est particulièrement illustrée à travers l'opposition entre les pratiques du ministère de l'économie d'une part et celles des ministères ingénieurs de l'autre. 
Le ministère de l'économie se caractérise en premier lieu par un investissement massif et récent dans l'outillage de gestion. Les outils accompagnant le renforcement de la qualité ou de la productivité, qu'ils prennent la forme d'outils de gestion ou d'applications informatiques, y sont particulièrement développés. Cette diffusion des outils de rationalisation de la production s'accompagne d'une mise sous pression du travail des agents qui est lisible dans les réponses des salariés. Le niveau de pression ressenti sur le travail à travers certains indicateurs est même tout à fait comparable à celui mesuré dans les secteurs du privé particulièrement exposés. Ces effets peuvent être cependant tout autant rapportés à la nature de l'activité au sein du ministère, et en particulier aux fonctions de traitement de dossiers individuels qu'à un style de réforme. En revanche, on constate qu'il n'est plus en tête pour l'usage des outils de gestion des ressources humaines permettant un traitement différencié des individus et que la différenciation dans la carrière des agents y est plus faible que dans les ministères ingénieurs ou dans les petits ministères rassemblés sous l'appellation d'administration générale, ce qui est plus difficile à expliquer par la nature des prestations effectuées.

Les ministères ingénieurs étaient présentés par tous les observateurs, dans les années 1990. comme modèles de la diffusion des outils de gestion du privé. Une telle primauté n'est plus visible dans le taux global d'équipement en outils de gestion, ces ministères étant tout simplement dans la moyenne. Elle demeure cependant pour ce qui concerne les outils associés aux ressources humaines. Elle demeure aussi en partie au travers d'une perception plus forte par les agents de leur possible différenciation. Ces différences sont cependant plus faiblement marquées que celles qui distinguent le ministère de l'économie. Le taux de diffusion de certains outils de ressources humaines peut être assez proche de celui observé au ministère de l'éducation nationale et la perception de la différenciation n'est pas très différente de celle observée dans les petits ministères. L'effet de modèle constitué à la fin des années 1980 s'est ainsi en partie estompé, mais il n'en demeure pas moins qu'il reste une trace dans la durée d'une orientation différente de la réforme.

La persistance d'un second modèle associé aux ministères ingénieurs conduit à nuancer deux positions de Philippe Bezes (2009) concernant la chronologie et la focalisation sur les «ministères généralistes». II semble qu'il y ait bien des mouvements plus longs d'appropriation des pratiques et de diffusion que ce que le découpage chronologique lié au balancement entre la mise sur agenda et le lancement des programmes de réforme laisse entendre, et il apparaît que des ministères spécialisés (les «ministères ingénieurs") ont occupé une position singulière essentielle à la compréhension de la réforme administrative, invitant à déporter l'attention des seuls ministères généralistes (intérieur/économie/plan/fonction publique).

\section{Réformer par les outils ou par les hommes?}

L'opposition entre «réformer par les outils» et «réformer par les hommes » peut être dans un premier temps lue comme une opposition entre 
catégories d'outils (la gestion de la production versus les ressources humaines). La « réforme par l'usager » (Strobel, Kaisergruber, 1996) qui est inscrite en particulier dans les outils de gestion incluant des engagements précis de prestation à l'égard des destinataires semble ainsi s'opposer dans l'association des outils de gestion à une «réforme par les agents » qui repose sur les outils de ressources humaines. Elle s'oppose aussi, dans les effets produits, à ce que l'on peut appeler une «réforme par le citoyen » dans laquelle ce dernier, via le parlement et le contrôle des dépense, devait mettre sous tension l'administration et dont on peine à mesurer des effets à travers l'enquête COI.

Mais l'opposition entre réformer par les outils et par les hommes se précise progressivement comme une opposition entre outils et pratiques. Ainsi, on voit émerger l'option d'une réforme techniciste qui repose en particulier sur des normes de qualité opposée à une pratique de recherche d'une meilleure adaptation du service ou d'une application plus complète de la règle par la capacité laissée aux agents d'apprécier les situations. De même, concernant le volet ressources humaines, ce ne sont pas les outils proprement dits comme l'existence d'un référentiel métier qui font la différence, mais bien une certaine attitude des responsables de ressources humaines.

\section{Conclusion}

\section{Des lectures complémentaires}

Au terme de cette discussion, les tenants des trois hypothèses présentées en introduction pourront trouver de l'eau à apporter à leur moulin. Les derniers développements sur la différence entre outils et pratiques illustrent directement l'opposition entre «méthodes " et «démarches » proposée par Sylvie Trosa (2010) et le fait que certaines finalités de la réforme risquent de ne pouvoir être atteinte par le seul déploiement technique d'outils de gestion. Cet inaboutissement ne peut cependant être considéré comme un blocage, le rythme de diffusion des outils de gestion témoigne au contraire de fortes transformations qui plaident bien pour l'hypothèse de la modalité discrète de réforme (Bezes, 2004) (Palier, 2004). C'est alors, par ce canal, une orientation particulière de la réforme qui s'impose. Ceci rejoint partiellement l'hypothèse proposée par Patrick Le Gales (2004). En effet, même si les données de l'enquête $\mathrm{COI}$ ne permettent que d'opposer deux figures avérées de la réforme, la connaissance que l'on peut avoir par ailleurs du rôle pris par le ministère de l'économie et des finances dans la gestion actuelle de la réforme et la place laissée aux ministères de l'Ecologie et de l'Agriculture après la RGPP permettent de penser que l'un des modèles a plus de raison de s'imposer que l'autre. En l'occurrence, il ne s'agit pas d'une réforme centrée sur les hommes, mais plutôt sur certaines formes de contrôle de l'activité. Mais ce renforcement du contrôle ne passe pas par les voies que Patrick Le Gales évoque. Les modalités du contrôle qui émergent ne sont pas celles associées au 'rendre compte', aux tableaux de bords et aux outils comptables qu'il met en avant, mais celles associées à la production et aux outils de qualité tels qu'analysés par Paul Hoggett (1996). C'est là bien un des apports de l'enquête COI, en complément quantitatif 
à de nombreuses observations qualitatives de sociologie sur le travail des fonctionnaires (Jeannot, 2008b) de confirmer que les transformations de l'administration se jouent largement autant dans l'organisation de l'activité quotidienne des services que dans la réforme budgétaire et le contrôle des coûts.

\section{Annexe méthodologique}

Le dispositif Changement organisationnel et informatisation (COI) associe une enquête a destination des salariés et une enquête à destination des employeurs. Un premier échantillonnage est établi sur la base des employeurs (entreprises ou administrations), l'échantillonnage des salariés est tiré parmi les employeurs enquêtés. Cette méthode permet de mettre en relation les réponses des deux questionnaires (enquête dite "couplée »).

Le volet fonction publique de l'enquête de 2006-2007 (COI-FP) a pris le parti de concentrer l'attention sur les fonctions administratives de l'Etat. Les enseignants, magistrats et le ministère de la Défense ont été exclus du champ de l'enquête COI-FP, en considérant que leur activité spécifique n'a pas d'équivalent dans le secteur privé marchand. Le questionnaire a été adapté pour correspondre aux pratiques de gestion du secteur public, dans le cadre d'un groupe de travail associant la DGAFP, le CEE et des chercheurs, auquel les auteurs ont participé, en s'appuyant sur une pré-enquête réalisée auprès d'une trentaine de responsables de services. Certains outils spécifiques au secteur public ont été ajoutés, certaines questions liées au contexte d'action ont été adaptées pour rendre compte de la spécificité de l'intervention publique. Un certain nombre de questions de l'enquête auprès des entreprises ont néanmoins été conservées, nous permettant d'établir quelques comparaisons de cadrage avec le secteur privé.

Les questionnaires ont été envoyés au cours de l'été 2007 aux responsables de services d'un échantillon de 400 directions centrales ou déconcentrées (unités disposant d'une délégation de signature, qui correspondent aux direction régionales et départementales de l'équipement, des impôts, du trésor, aux rectorat ou établissement d'enseignement secondaire ou supérieurs de l'éducation nationale,etc. et aux directions d'administration centrale), stratifié selon le ministère et la taille du service (dans le cas de l'éducation nationale -resp. de la justice-, les enseignants -resp. les magistrats- ont été retirés de la base pour le calcul de la taille de la direction). Environ 300 directions ont répondu. Les questionnaires ont dans la plupart des cas circulé dans les services (en particulier entre le secrétariat général et les services informatiques) ce qui garantit la compréhension de certains libellés d'outils informatiques ou de gestion.

L'échantillon de l'enquête salarié (1000 répondants) a été tiré dans les directions retenues pour l'enquête employeur, ce qui permet de connaître pour un agent donné les outils de gestion mobilisés dans son service (mais ils ne le concernent pas toujours directement) et d'établir des corrélations entre les pratiques de gestion et la perception par les agents de leur travail. 
Le questionnaire à destination des salariés est strictement identique à celui utilisé pour les salariés des entreprises. II comprend des questions, le plus souvent factuelles, à propos de la vie quotidienne au travail. La pondération des réponses des employeurs et des salariées permet d'estimer les résultats sur l'ensemble de la population, en corrigeant les déformations liées au mode d'échantillonnage à deux niveaux et à la non réponse.

Le traitement des données propre au présent article repose sur deux regroupements, des ministères et des outils.

La taille réduite de l'échantillon d'employeurs ne permettait pas de traiter isolément tous les ministères. Après avoir isolé les ministères de l'économie et des finances et la partie administrative du ministère de l'éducation nationale qui constituent des ensembles de taille suffisante, les premiers traitements ont mis en évidence la spécificité de la police par rapport au reste du ministère de l'intérieur et celle des ministères de l'équipement et de l'agriculture par rapport à l'ensemble des autres ministères. Nous avons ainsi créé une catégorie "police», une catégorie "ministères ingénieurs" regroupant l'équipement et l'agriculture et une catégorie «administration générale» regroupant le reste de l'échantillon.

Concernant les outils nous avons privilégié, parmi les instruments de gestion traités dans le questionnaire, 4 catégories: instruments de la qualité, instruments d'organisation de la production, technologies de l'information et de la communication et outils de ressources humaines. Les taux d'équipement ont été établis à partir de cette segmentation et des indicateurs synthétiques d'équipement ont été construits en cumulant le nombre d'équipements par direction dans chacune des catégories retenues.

- Qualité (5 outils): certification ou labellisation de la qualité des services rendu (NF, etc..), Etablissement ou suivi d'une charte de qualité (charte Marianne, FQP, etc..) ; Engagement contractuel à traiter un dossier dans un délai limité ; Enquêtes de satisfaction des usagers ; Contrôles ou audits de la qualité de service.

- Production (6 outils): Certification ou accréditation portant sur le système qualité, les compétences techniques ou la sécurité (ex ISO 9001, EAQF, etc..) ; Audit sur les coûts et les procédures ; Méthodes formalisées de résolution de problèmes, analyse de la valeur... ; Flux tendus, délais de production impératifs ; Equipes de travail autonomes (par exemple dans le cadre d'un management de projet).

- Ressources humaines (6 outils): Gestion prévisionnelle (GPRH ou GPEEC) ; référentiel de compétences ou de métier ; Système d'affichage des postes vacants à l'ensemble du personnel ; Dispositif de mutation sur fiche de postes profilés ; Dispositif d'aide à l'évolution des métiers ou des fonctions (bilan de compétences) ; Dispositif de conseil de carrière ou d'aide à la préparation des concours. 
- Technologie de l'information et de la communication (TIC) (11 outils) : Information accessible au public sur le web : Délivrance de services en ligne (e administration); Possibilité pour les usagers de suivre leur dossier sur internet; Outils de gestion informatique intégrée de la relation usagers (CRM); Bornes télématiques ou guichets automatiques; Outils d'archivages et de recherche automatiques des données (GED); Outil d'analyse des données; Outils de travail collaboratifs (groupware, visioconférences) ; Outils d'interfaçage de bases de données; Outil d'automatisation des processus (Workflow); Progiciels de gestion intégrée (ERP).

\section{BIBLIOGRAPHIE}

Barouch G. (1994), « Un deuxième souffle pour la modernisation des administrations d'Etat : analyse et proposition », Politiques et management public, vol 12, n4, p. 143-156.

Bartoli A. (1997), « Les outils du management public, fausses pistes et vrai défi » in : Huron D., Spindler J., Le management public en mutation, L'Harmattan.

Bartoli A. (1997), Le management dans les organisations publiques, Dunod.

Bezes P. (2004), « Rationalisation salariale dans l'administration française, un instrument discret » in : Lascoumes P., Le Galès P., Gouverner par les instruments, Presses de science po, p. 71-122.

Bezes P. (2009), Réinventer l'Etat, Les réformes de l'administration française (1962-2008), Puf.

Bouckaert G. (2003), « La réforme de la gestion publique change-t-elle les systèmes administratifs ", Revue française d'administration publique, $n^{\circ} 105-106$, p-39-54 (p. 52).

Boussard V. (2001), "Quand les règles s'incarnent. Les indicateurs prégnants ", Sociologie du travail, vol. $43, n^{\circ} 4$, p. 533-551.

Brunsson N. (1982), The organization of Hypocrizy, The decision and action in organizations, John Wiley and son.

Chevalier Y. (2006), "La réforme budgétaire et la gestion des ressources humaines : quelles conséquences pour la fonction publique? ». L'Actualité juridique. Droit administratif (AJDA), n 10, 13 mars, p.523-531.

Cochoy F., Garel J. P., De Terssac G. (1998), «Comment l'écrit travaille l'organisation, le cas des normes Iso 9000 ", Revue française de sociologie, vol 39, n4, p. 673-699.

Emery Y., Giauque D. (2007), Dilemmes de la GRH publique, LEP.

Giauque D. (2003), La bureaucratie libérale, L'harmattan.

Gibert P. (1986), «Management public, management de la puissance publique », Politiques et management public, vol 4, n², p. 89-123. 
Gibert P., Thoenig J. C. (1993), « La gestion publique : entre l'apprentissage et l'amnésie ", Politiques et management public, vol 11, n¹, p. 3-21.

Greenan N. et Hamon-Cholet S. (2000), «Les salariés industriels face aux changements organisationnels en 1997 ", Premières Synthèses, DARES, mars, $n^{\circ}$ 09-3.

Greenan N., Mairesse J. (dir.) (2006), « Réorganisations, changements du travail et renouvellement des compétences ", Revue économique, vol. 57, $\mathrm{n}^{\circ}$ 6, novembre p. 1177,1203

Guillemot D. et Kocoglu Y. (2010), «La diffusion des outils dans les entreprises françaises, une approche synthétique ", Réseaux, vol 28 -162 p. 167-198.

Hoggett Paul (1996), « New modes of control in the public service », Public administration, vol 74, p. 9-32.

Jeannot G.(2008a), « Réforme de la fonction publique et réorganisation de l'Etat », Esprit, décembre, p. 94-109.

Jeannot G. (2008b), « Les fonctionnaires travaillent-ils de plus en plus ? Un double inventaire des recherches sur l'activité des agents publics ", Revue Française de science politique, $n^{\circ} 1$, p. 123-140.

Johnson T., Kaplan R. (1987), Relevance lost, The Rise and Fall of Management Accounting, Harvard business school press.

Kuhlmann S., Bogumil J., Grohs S. (2008), « La modernisation de l'administration publique locale en Allemagne : succès ou échec de la nouvelle gestion publique ", Politiques et management public, vol. 26, $\mathrm{n}^{\circ} 4$, p. 25-43.

Lascoumes P., Le Gales P. (2004), Gouverner par les instruments, Presses de science po.

Le Gales P. (2004), « Contrôle et surveillance, la restructuration de l'Etat en Grande Bretagne » in : Lascoumes P., Le Gales P. (2004), Gouverner par les instruments, Presses de science po, p. 237-272.

Lemarchand Y. (1993), Du dépérissement à l'amortissement : enquête sur l'histoire d'une concept et de sa traduction comptable, Ouest éditions.

Moisdon J. C. (1997), Du mode d'existence des outils de gestion, Paris, Seli Arslan.

Ogien A. (2002), L'esprit gestionnaire, éditions de l'EHESS.

Maugeri S. (2002), «Pour une sociologie de la gestion et de ses dispositifs », Terminal, $n^{\circ} 87$, p. $13-29$.

Palier B. (2004), « La politique des retraites en France » in : Lascoumes P., Le Gales P., Gouverner par les instruments, Presses de science po. p. 273-300.

Paucelle J. L. (2007), «La malléabilité organisationnelle des TIC », Revue française de gestion, $n^{\circ} 172$, p. 105-115.

Poinsard R. (1987), « La modernisation de la gestion administrative : un bilan ", Politiques et management public, vol5 $\mathrm{n}^{\circ} 2$, p. 21-63. 
Un bilan quantitatif de la «modernisation de la gestion » de l'État

Rainey H.G., Bozeman B. (2000), "Comparing public and private organizations: empirical research and the power of a priori", Journal of public administration research and theory, vol 10, $n^{\circ} 2$, p. 447-469.

Rangeon F. (1992), « la notation des fonctionnaires en question », Politiques et management public, vol10, $n^{\circ} 2$, p. 193-211.

Segrestin D. (2004), Les chantiers du manager, Paris, Armand Colin.

Silicani J. L. (2008), Livre blanc sur l'avenir de la fonction publique. Faire des services publics et de la fonction publique des atouts pour la France, Paris, La Documentation française.

Strobel P., Kaisergruber D. (1996), "Services publics fin de siècle ", in : Grémion C. Fraisse R., Le service public en recherche, quelle modernisation? La Documentation française, p. 21-38.

Trosa S. (2010), « Donner sens aux méthodes de modernisation de l'administration ", Revue française d'administration publique, $\mathrm{n}^{\circ} 135$, p. 533-548.

Trosa S. (2004), «Payer les fonctionnaires au mérite ?» Sociétal, $n^{\circ} 44$, p. 32-36.

Warin P. (2002), Les dépanneurs de justice, Les «petits fonctionnaires» entre qualité et équité, Paris, LGDJ.

Weber M. (1991), Histoire économique, Paris, Gallimard. 
